\title{
Time to death and its predictors among adult with drug-resistance tuberculosis patients, in Eastern and East-Central Ethiopia, 2012-2018: A retrospective cohort study
}

\section{Tsige Gebre}

Debre Markos University

Mulatu Ayana Hordofa

Ambo University

Molla Yigzaw Birhanu

Debre Markos University

Melkamu Siferih

Debre Markos University

Cheru Tesema Leshargie ( $\nabla$ chertesema@gmail.com )

Debre Markos University

\section{Research article}

Keywords: Drug-resistant tuberculosis, Predictors, Retrospective follow-up, Time to death, Eastern Ethiopia

Posted Date: October 10th, 2019

DOI: https://doi.org/10.21203/rs.2.15941/v1

License: (c) (i) This work is licensed under a Creative Commons Attribution 4.0 International License.

Read Full License 


\section{Abstract}

Background Drug-resistant tuberculosis (DRTB) is becoming a global public health problem in developing country including Ethiopia. It poses a greater challenge to the tuberculosis control program. Tuberculosis drugs namely; rifampicin and isoniazid, were the two most effective anti-tuberculosis drugs for which the agent become resistant. Understanding the survival time and the predictors of DRTB patients would be helpful to policy-makers and health practitioners in Ethiopia. However, there is a limited previous study on the aspect. Therefore, this study aimed to estimate the survival time and predictors of adult DRTB patients, in Eastern and East-Central Ethiopia.

Methods A retrospective follow-up study was conducted in the Eastern and East-Central part of Ethiopia among adult drug resistance-tuberculosis patients from 1st September 2012 to 30th August 2017. The checklist was used to retrieve information among a total of 362 drug-resistant tuberculosis patients. Kaplan Meier curve method was used to estimate the median survival time with its interquartile range and risks. Multivariable Cox proportional regression modelling was used to investigating predictors of survival time. Hazard ratio with $95 \% \mathrm{Cl}$ was used to report the findings of regression modelling.

Result A total of 362 participants were followed for 132,801 person day observation. During the follow-up period, there were 55 deaths with the overall incidence rate of 4 participants per 10,000-person day observation $(95 \% \mathrm{Cl}: 3.18,5.39)$. DRTB related death was higher among patients who had weight loss (AHRa: 9.0, 95\% Cl:2, 20.5), pulmonary with extra-pulmonary DRTB (AHR:10, 95\% Cl:3.3, 16), HIV coinfection (AHR:4, 95\% Cl:2.1,7.5) and comorbidity (AHR:4.40, 95\% Cl:1.7, 11), patients with BMl<18.5 (AHR:0.22, 95\% Cl:0.076, 0.63) were less likely to die of MDR-TB. BMK 18.5 patients with a history of relapse (AHR: $4.2,95 \% \mathrm{Cl}: 1.9,9)$ and after the failure of re-treatment (AHR: $6.3,95 \% \mathrm{Cl}: 2.6,13$ ) were predictors of DRTB death.

Conclusion To summarize, the survival time of patients with drug-resistant tuberculosis was low. DRTB related death was higher among patients with weight loss, people with extra-pulmonary, HIV co-infection, comorbidity, and history of relapse.

\section{Background}

Drug-resistant tuberculosis (DRTB) became a global public health problem. It is an emerging, especially in developing country posing a greater challenge for the national tuberculosis control program. It is a type of tuberculosis (TB) which is resistant for a drug like rifampicin and isoniazid, the two most powerful anti -TB first-line drugs [ 1-4]. Now a day, the low and middle-income countries are significantly suffering from DRTB and its complications. Moreover, in a country where HIV infection is epidemic, the occurrence of DRTB reported as increasing. One-third of the world population is infected by TB and an estimated 1.5 million death occurred each year [ 5, 6]. The highest DRTB mortality rate was reported from Africa and Southeast Asia [ 7, 8]. Accordingly, Ethiopia is among the 
30 DRTB high-burden countries, with an estimated 5800 cases reported in 2016 with the prevalence rate of $2.7 \%$ among new cases and $14 \%$ among previously treated patients [ 8 , 9]. Half of the DRTB treatment has a poor outcome as compared with non-drug resistance TB (10\%). Poor treatment response is what lengthens treatment since people will continue to receive treatment if they do not culture-convert [ 10].

HIV infection, undernutrition and limited access to a well-equipped health facilities were identified as common factors extensively worsen the treatment outcome DRTB [ 11]. Moreover, population growth and changes in age structure account for $79.5 \%$ of the predicted increase for new cases. Age-specific incident rates in Sub-Saharan Africa are increasing due to the HIV epidemic and account for the remaining 20.5\% [ 12]. Similarly, age (being older) [ 13-15], Co-morbidities like HIV infection, HIV drug resistance, Diabetes mellitus and chronic kidney disease and undernutrition, cavitary disease [ 13, 15-20], CD4 count (<200 cells/ul) [ 21], gender (being male) [ 14], residence (rural living), unemployment and lower educational level [ 15] and patient life-style like alcohol abuse [ 17, 18], smoking [ 17-20], and drug use [ 18] were frequently reported as a common predictor for the occurrence of DRTB.

Even with, the notable sound effects of directly observed therapy strategies (DOTS) implemented to reduce, control and also increase the positive treatment outcome of tuberculosis the rate of DRTB become increased and prone to be the leading problem of Ethiopia [ 22-24]. Lack of social support and poor patient Cohort recognized a significant factor for the increased rate of MDR-TB. These patients might also have a chance to transmit the disease in case of bacterial reversion and leads to poor prognosis [25]. Therefore, this study aimed to assess the time to death and its predictors among adult DRTB participants in Eastern and East-Central Ethiopia, 2018

\section{Methods}

\section{Study Design, Study Area, Period and Populations}

An institution-based retrospective cohort study was conducted for the duration of first September 2012 to 30th August 2017 in Eastern and East-Central Ethiopia health facilities namely, Dil Chora referral hospital, Amir Nur health center, and Hailemariam referral hospital. Dil Chora referral hospital is found in Dire Dawa town 525 kilometres from Addis Ababa, the capital city of Ethiopia in a northwest direction. Based on the 2015 population projection, Dire Dawa had a total population of 440,000. The second study setting, Amir Nur health center, is found in Harari town which is located at a distance of $526 \mathrm{~km}$ from Addis Ababa in southeast Ethiopia. Based on the 2015 population projection, Harari had a total population of 232,000 . The third study setting, Hailemariam referral hospital is found in Adama town, which is $99 \mathrm{kms}$ from Addis Ababa in East-Central Ethiopia. Based on the 
2015 population projection, this city had a total population of 324,000 . These health facilities serve for more than 17 million people and care for the largest proportion of DRTB patients in the country. All adult DR-TB patients from first September 2012 to $30^{\text {th }}$ August 2017 in Eastern and Central Ethiopia were included in the study.

\section{Sample size determination and sampling procedure}

The required sample was determined using Stata 14 version statistical software based on the assumption adjusted hazard ratio (AHR) for clinical complication, 1.9, event 82 and the probability of event 0.29 [ 21] and adding $10 \%$ for the incomplete record. Based on these assumptions we found a sample of 377. Therefore, 377 DRTB patients were included in this study, which 167 were from Hailemariam hospital, 194 from Dil Chora Referral Hospital and 16 from Amir Nur health center. After excluding incomplete patient medical records, the total number of DRTB patient records in the three health facilities was 362. Since it was manageable, all records were included in the study.

\section{Operational Definitions}

- Time to death: for this study time to death was calculated by subtracting date of treatment initiation $\left(t_{0}\right)$ from date of death occurred $\left(t_{1}\right)$.

- Cured: is defined as patients without evidence of failure and three or more consecutive cultures taken at least 30 days apart are negative after the intensive phase.

- Completed treatment: A patient who completed the anti-TB regime for 18 months or over 12 months.

- Poor treatment outcome: is defined as unsuccessful treatment leading to death, TB relapse, loss to follow up treatment, or fail to complete treatment regimen or treatment interruption.

- Death: is defined as a patient who died during the treatment respective of the cause (i.e., death after the end of DRTB treatment course is not included or considered by this study).

- Failed treatment: is defined as a smear-positive patient who remained smear- positive by the end of the intensive phase, or bacteriological reversion in the continuation phase after conversion to negative.

- Censored: when the outcome of interest has not been observed for an individual during the treatment course period. DRTB who cured, treatment completed, lost to follow up, transfer out with unknown treatment results and those on treatment were treated as censored.

\section{Data extraction checklist and course of action}


An English version data extraction checklist was developed from related literature and patient registration book. The checklist contains characteristics like socio-demographic data, clinical characteristics, HIV co-infection, co-morbidities, nutritional status, site of involvement, radiological findings. Patient medical records: including registration and monitoring records were used as a source of data.

\section{Data quality control}

To maintain the quality of data the following activities were conducted: the data extraction checklist was pretested on other but similar population. Moreover, a one-day training was given for the data collectors (nurses with first-degree holder working in TB clinics) focusing on the data extraction producers, checklist, and objectives of the study. Furthermore, close and routine supervision was done by the investigators of the study. Addition, the authors and an experienced data clerk entered and cleaned the data before the analysis.

\section{Data Processing and analysis}

The collected data were entered to EpiData version 4.2 and further analyses were done using stata version 14 statistical packages. Survival curves were compared between different exposure groups using a log-rank test (Chi-square test with $1 \mathrm{df}$ under $\mathrm{H}_{\mathrm{o}}$ ). Survival trend over the follow-up time was computed using the Kaplan Meier (KM) method and covariates significant in Bivariable analysis at $\mathrm{p}<0.25$ were fitted to multiple Cox regression model to show the independent predictor of death. After fitting the Cox proportional hazard regression model, the adequacy of the fitted model to the survival data was checked using Cox-Snell residuals with the Nelson-Aalen cumulative hazard graph. An assumption to Cox-proportional hazard model that is the hazards remaining proportionately constant was checked using graphical representation (Log-log plot) and Schoenfeld residuals. In addition to this, time varies covariates were checked and all of the covariates were constant overtime. Hazard ratio with its $95 \% \mathrm{CI}$ and the p-value was used to measure the association of dependent and independent variables.

\section{Results}

\section{Socio-demographic Characteristics}

A total of 362 medical records patients with drug resistant tuberculosis (DRTB) were reviewed giving a response rate of $96 \%$. The median age of the participants were 31 (IQR= 24-41) years old. Of the 362 patients included in this study, 193 (53.31\%) were males. Two hundred eighty (77.35\%) patients were Oromo, 49 (13.54\%) were Somali, 26 (7.18\%) were Harari and 7 (1.93\%) were Amhara. Slightly more than have, 214 (56.8\%) of the patients were from rural residence. 


\section{HIV Co-infection and other Co-morbidities}

Of all DRTB patients, 76 (20.99\%) were HIV positive. Among these, 35 (46.05\%) were diagnosed. Diabetes mellitus was the most prevalent (50\%) followed by chronic kidney disease and hypertension. Congestive heart failure, chronic liver disease, hepatitis, and epilepsy were less prevalent (Table 1).

Table 1: Human immuno deficiency virus co-infection and other medical co-morbidities in DRTB patients in Eastern and East-central Ethiopia, 2012-2018

\begin{tabular}{llll}
\hline Variable (N=362) & Categories & Frequency & Percent \\
\hline HIV co-infection & Positive & 76 & 21.0 \\
& Negative & 286 & 79.0 \\
\hline \multirow{5}{*}{ Other co-morbidities } & Diabetes mellitus & 11 & 50.0 \\
& Chronic kidney disease & 4 & 18.2 \\
& hypertension & 4 & 18.2 \\
& CHF and Hepatitis & 1 & 4.5 \\
& Liver disease & 1 & 4.5 \\
& Epilepsy & 1 & 4.5 \\
\hline
\end{tabular}

\section{Nutritional Status}

The median baseline weight was $49(\mathrm{IQR}=41-57)$ kilograms. The median BMI was 16.85 (14.7-19.4). Of all the participants, 290 (80.11\%) of them experienced weight loss during the treatment.

\section{Clinical Characteristics DRTB Participants}

More than half of the patients with drug resistant tuberculosis (225/362, 62.15\%) were newly diagnosed as DRTB while the remaining patients were previously treated with antiTB medications. Previously treated patients includes 62 (17.13\%) relapses, 21 (5.8\%) after lost to follow up, $32(8.84 \%)$ after the failure of first treatment, 22 (6.08\%) after the failure of re-treatment (Figure 1).

\section{Clinical Presentation of DRTB Patients}

The majority of the DRTB patients were presented at least three of the following clinical presentations: cough (359/362, 99.17\%), night sweating (349/362, 96.41\%), loss of appetite (345/362, 93.3\%), chest pain $(54 / 362,14.9 \%)$ and fever (228/362, 62.98\%). Vomiting, headache, abdominal distension, haemoptysis, neck stiffness, diarrhoea, joint pain, and carcinogenic shock were prevalent in smaller proportions.

\section{Laboratory Results and Radiologic Findings}


Among all medical record, 323 (89.23\%) were smeared positive during diagnosis while 333 (92.27\%) were culture positive. The majority of the DRTB patients, 347 (95.86\%) were diagnosed with pulmonary DRTB, whereas 4 (1.1\%) with only extra-pulmonary DRTB and 11 (3.03\%) with both pulmonary and extra-pulmonary DR-TB. Among these complications, pneumonia was the commonest $(30 / 33,91 \%)$ followed by pneumothorax (3/33, 9\%). According to the chest x-ray report, 190 (62.5\%) had a cavity, 97 (31.91\%) had infiltration, and 17 (5.59\%) had bronchiectasis.

\section{Drug Susceptibility Testing (DST) Result}

Based on drug susceptibility testing (DST) results, 307 (84.81\%) DRTB patients had developed resistance to Rifampicin, 39 (10.77\%) to both Rifampicin and Isoniazid and 16 (4.42\%) to Rifampicin, Isoniazid, and another drug.

\section{Adverse Effects of the Drug}

Gastritis (78/107, 72.90\%), nausea and vomiting (32/107, 29.91\%), Hypokalemia (73/107, $68.22 \%)$, and Ototoxicity (21/107, 19.63\%). Renal toxicity, blurred vision, Arthralgia, psychotic symptoms, hepatotoxicity, Anemia, Hypovolemic shock and vomiting were less prevalent (Table 2).

Table 2: Drug adverse effects in DRTB patients in Eastern and East-central Ethiopia, 20122018

\begin{tabular}{lll}
\hline Adverse Effects & Frequency & Percentage (\%) \\
\hline Gastritis & 78 & 72.9 \\
Hypokalemia & 78 & 68.22 \\
Nausea and vomiting & 32 & 29.91 \\
Ototoxicity & 21 & 19.63 \\
Renal toxicity & 8 & 7.48 \\
Psychotic symptoms & 4 & 3.73 \\
Arthralgia & 4 & 3.73 \\
Blurred vision & 4 & 3.73 \\
\hline
\end{tabular}

\section{Time to death of DRTB Patients}

A total of 362 DRTB patient medical records were followed for different exposure periods contributing a total 438 median times with a range of 1 to 980 days follow-up time over 32,801 person-day observations. During the follow-up periods, there were 55 (15.2\%) deaths that make the overall incidence rate of 4 patients per 10,000 person-day observation 
(95\%CI: 3.18 - 5.39). The median time to death was not estimated well due to high observational censored. In addition to this, since the largest observed analysis time was censored, the mean survival time was underestimated. Therefore, both the restricted and extended mean were used to determine the survival time. The restricted mean survival time was 26.6 (95\% CI=25.3, 28.7) months and the extended mean (survival probability) at the end of the follow-up period was $73.79 \%$ (95\% CI: 57.96\%, 84.4\%). Greater proportions of deaths were registered during the first five months of treatment. DRTB patients survival time at the end of $1^{\text {st }}, 2^{\text {nd }}, 3^{\text {rd }}, 4^{\text {th }}$ and $5^{\text {th }}$ months were $95.77 \%, 93.44 \%, 91.04 \%, 89.48 \%$, and $88.47 \%$ respectively (Figure 3 ).

\section{Survival function of a predictor variable}

Cochran-Mantel Haenszel log-rank test was used to check the equality of survival curves for categorical predictor variable. The result indicates that there was a significant difference in the survival function of the categorical variable particularly HIV status. The hazard dearth rates for DRTB patients who were HIV positive at the time of treatment were significantly shorter compared to their counterparts (Figure 4).

\section{Treatment Outcome}

Concerning about treatment outcome, this study found that about half, 180 (49.7\%) of DRTB patients were completed and cured (Table 3). In addition, after fitting the Cox proportional model, the adequacy of the model was checked by Nelsen Aalen cumulative hazard with Cox-Snell residuals (supplementary file 1).

Table 3: Treatment Outcome of DRTB patients in Eastern and East Central Ethiopia, 20122018

\begin{tabular}{llll}
\hline Treatment outcome & Frequency & Percentage (\%) \\
\hline 1 & Cure/Complete & 180 & 49.7 \\
2 & Death & 55 & 15.19 \\
3 & Lost to follow up & 30 & 8.29 \\
4 & Transfer out & 14 & 3.87 \\
5 & On treatment & 83 & 22.93 \\
\hline
\end{tabular}

\section{Predictors of Mortality}

From all; (i.e., sex, age residence, ethnicity, occupation, marital status, educational status, economic status, baseline weight, site of involvement, HIV reactive, comorbidity, previous TB treatment, BMI, more than isoniazid and rifampicin resistance) fitted during the 
bivariable analysis only age, baseline weight, site of involvement, HIV reactive, comorbidity, previous TB treatment, BMI, more than isoniazid and rifampicin resistance were found association with DRTB death. After controlling the effects of confounding variables, in multivariable Cox proportional analysis, only seven variables were found to be predictors of DRTB deaths. The result of the multivariable analysis showed that DRTB patients who loss weight during treatment were nine (AHR: 9, 95\%CI: 2, 20.5) times more likely to die compared to those without weight loss. Those patients with BMI $\geq 18.5$ were $78 \%$ less likely to die. Patients whose baseline weight of $\geq 45 \mathrm{~kg}$ were $60 \%$ less likely to die compared with $<45 \mathrm{~kg}$. DRTB patients who had previous TB treatment registered as relapse were four (AHR: 4.2, 95\%CI: 1.9, 9.0) times more likely to die compared to new DRTB patients. Whereas patients registered as after failure of re-treatment were six (AHR: 6.3, 95\%CI: 2.6, 13) times more likely to die compared to new DRTB patients. Patients who had both pulmonary and extra-pulmonary DRTB were ten (AHR: 10, 95\%CI: 3.3, 16) times more likely to die compared to their counterparts. Patients with HIV co-infection were four (AHR: 4, 95\% CI: 2.1, 7.5) times more likely to die than HIV negative patients. DRTB patients who had other medical problems (co-morbidities) were about four (AHR: 4.4, $95 \% \mathrm{CI}: 1.7,11$ ) times more likely to die compared to patients without co-morbidities (Table $4)$.

\section{Discussion}

Worldwide, the emergence of DRTB poses a greater public health problem to tuberculosis control programs [ 26]. This institution based, retrospective Cohort study sought to estimate the time to death among DRTB patients and its predictors in the Eastern and Eastcentral region of Ethiopia.

Our current study found that the overall incidence rate was 4 patients per 10,000 personday observation (95\%CI: 3.18, 5.4). This study compares well with study findings reported by St. Peter specialized TB hospital in Addis Ababa, Ethiopia which was 3.6 per 10,000 person-days [ 19]. 
In another way, our finding is higher than the study conducted in southern Ethiopia which was 2 patients per 10,000 person-day [27]. This difference might be due to the difference in the sample size variation. In this study, a large number of patients register in five years were followed compared to the previous studies conducted in southern Ethiopia.

In this study, both the extended and restricted mean were used to determine survival status. Therefore the overall mean survival probability at the end of the follow-up period was 73.79\% which lends support to a study conducted at St. Peter hospital (78.95\%) [ 19]. In another way, this finding on mean survival probability is lower than the study conducted in southern Ethiopia (88\%) [ 27]. The possible explanation for this might be due to the difference in the follow-up periods. That means as the follow-up period becomes longer, the probability of the event becomes decreased.

This study also identified a predictor of mortality among DRTB patients. In this study, DRTB who experience weight loss during treatment was 9 (AHR: 9, 95\%CI: 2.0-20.5) times more risk to die as a compared with counterpart. This finding goes in good agreement with the study reported by southern Ethiopia, Bulgaria and Lima, Peru [ 14, 27, 28]. The possible explanation for this may be, DRTB participants experience severe gastro-intestinal intolerance (nausea, vomiting and gastritis) and drug toxicities during treatment that causes malnutrition and this may reduce the survival probability of the participants. This is scientifically supported that anti-TB drugs have serious adverse effects including nausea, vomiting and electrolyte disturbance which leads to poor prognosis [ 29].

The current study also looked at predictors of mortality among DRTB patients of included participants and undernutrition was identified as a predictor for mortality among DRTB participants. DRTB clients with BMI <18.5 were 78\% (AHR: 0.22, 95\%CI: 0.076-0.63) more risk to die compared with the counterpart. This finding corroborates with the study findings' reported in Lima, Peru and Canada[ 14, 28]. The possible explanation might be that DRTB causes secondary malnutrition as the participants' loss of appetite, nutrient, and micro-nutrient. This is also evidenced by that mal-absorption and altered metabolism 
resulted in poor survival. In turn, undernutrition could lead to secondary immunodeficiency which exacerbates poor survival of DRTB participants [ 30].

Moreover, HIV status was found as a predictor among patients of DRTB mortality. DRTB patients mortality who are positive for HIV were 4 (AHR: 4, 95\%CI: 2.1-7.5) times more risk as compared with their counterpart. Our findings lent support to the findings reported in Vietnam, Lithuania, St. Peter TB Specialized hospital, Lima-Peru, United Kingdom and Egypt [ 14, 15, 30-33]. The high mortality rate of DRTB patients among HIV positive participants might be due to the synergistic effects of the two co-infection HIV and DRTB. Patients receive treatment for a minimum of two years. The patients might experience serious drug adverse effects and toxicities due to the high burden of pills. As a result, patients could experience non-adherence of the treatment and end-up with poor outcome. In turn, the diagnosis of DRTB in HIV positive patients is more difficult as may be confused with other pulmonary or systemic infection and the rate of smear negativity is also high in HIV patients. This can result in misdiagnosis or delay in diagnosis and leads to higher morbidity and mortality [ 3,34$]$. Diabetes is a common comorbidity in DRTB patients, and also similar in this study. Diabetes can worsen the clinical course of DRTB and DRTB can also worsen the glycaemia control of diabetes. Anti-TB drugs and diabetic medications may have overlapping toxicities like peripheral neuropathy which leads to death [ 31, 32].

This study also found that previously TB treated DRTB patients, had a history of treatment relapse and re-treatment failure were significant predictors of death. This is also agreed with study conducted in Estonia [ 18, 33, 35, 36]. The possible reason for this might be Mycobacterium tuberculosis acquires further resistance to anti-TB drugs by spontaneous mutations and changed to the most potent form of bacteria which is called Beijing Lineage. Unless appropriate drugs are administered, this could lead to unfavorable treatment outcome. This is also scientifically supported that Beijing Lineage Mycobacterium strain of bacteria overproduces Triglycerides which leads to lethal disease [ 17, 37].

In addition, the site of DRTB involvement also found to be predictors for mortality. Pulmonary with extra-pulmonary DRTB was found to be a predictor of DRTB patients 
mortality that proves report in Lima Peru [ 16]. The possible explanation for this might be the probability of delayed diagnosis in patients with extra-pulmonary DRTB due to the similarity of clinical presentations with other diseases. In fact Pulmonary with extrapulmonary DRTB further complicates the survival of the participants due to the extensive damage of the lung and other organs [ 38].

\section{Limitation of the Study}

This study included all deaths recorded during the follow-up period. The cause of death was not recorded as the data were extracted from medical records. Several socio-demographic variables namely family size, employment, educational status, and income, and substance abuse, radiologic were missed due to the incompleteness of the records.

\section{Conclusions And Recommendations}

To summarize, the current study found that survival time of patients with drug-resistant tuberculosis was unacceptably low. Baseline weight, weight loss during treatment, lower BMI, HIV co-infection, comorbidity, pulmonary with extra-pulmonary DRTB and previously TB treatment were identified as predictors for death among patients with drug resistant tuberculosis. As a recommendation, health professionals together with Governmental and Non-governmental organizations like Heal-TB should take into account the nutritional status of patients with drug-resistant tuberculosis. Moreover, early detection, initiation of the treatment and appropriate follow-up should be undertaken by the health professionals.

\section{Abbreviations}

BMI: Body Mass Index

HIV: Human Immunodeficiency Virus

DRTB: Drug Resistant Tuberculosis

WHO: World Health Organization

\section{Declarations}

\section{Ethics approval and consent to participate}

Ethical clearance was obtained from Debre Markos University Ethical Review Committee. Then permission letter was taken from the three Hospitals' administrative bodies. Personal 
identifiers that were recorded on medical records were not used.

\section{Consent for publication}

“Not applicable”

\section{Availability of data and materials}

"The data set would not be shared to anyone in order to protect the participants' identities"

\section{Competing interests}

The authors declared that there was no conflict of interest.

\section{Funding Body}

"Not Applicable"

\section{Author's contribution}

TG: designed and developed the study, performed analysis and interpretation of data. CT and MA, MY and SM: supervised and guided the design and development conception, analysis, interpretation of data and made critical comments at each step of research. TG and CT drafted the manuscript. All authors read and approved the final Manuscript.

\section{Acknowledgment}

We would like to express our deep gratitude to data collectors and supervisors for their timely submission of the collected data. We would like also to forward our gratitude for Dil Chora Referral Hospital, Amir Nur health center and Hailemariam Referral Hospital administrative bodies who permit us to conduct this study. Authors want also to thank Debre Markos University for arranging internet and library services. Last but not least, we would like to forward our deepest gratitude to Kedir Yimam Ahimed (a BSc, MPH, and assistant professor in public health and Ph.D. Candidate in public health, in western Sydney University, Australia) for his extensive language and other writing, typology and technical error edition.

\section{References}


1. World Health Organization (WHO): Multidrug and Extensively Drug-Resistant Tb (M/XDR-Tb) 2010 Global Report on Surveillance and Response,. 2010.

2. World Health Organization (WHO): Global tuberculosis report Geneva: 2013.

3. World Health Organization (WHO): WHO guidelines for the programmatic management of drug-resistant tuberculosis, guideline. 2014.

4. World Health Organization (WHO): Guidelines for surveillance of drug resistance in tuberculosis 5th Edition,. 2015.

5. Dolin PJ, Raviglione MC, Kochi A: Global tuberculosis incidence and mortality during 1990-2000,. 1994.

6. Dye C, et al.: Global burden of tuberculosis: estimated incidence, prevalence, and mortality by country. Jama ,. 1999, 282(7):677-686.

7. World Health Organization (WHO): Global Tuberculosis Report 20th edition,. 2015.

8. World Health Organization (WHO): Global tuberculosis report 2017. Geneva, Swizerland:. WHO press 2017.

9. Federal Ministry of Health of Ethiopia (FMOH): Tuberculosis prevention and control programme: special issue for world TB day. 2011, 3(1):17-37.

10. Federal Ministry of Health (FMOH): Guidelines on Programmatic Management of Drug Resistant Tuberculosis in Ethiopia, Guideline, . 2013.

11. Center for Strategic and International Studies: As Ethiopia Moves toward Tuberculosis Elimination, Success Requires Higher Investment, Report, . 2016.

12. Alami NN, Yuen CM, Miramontes R, Pratt R, Price SF, Navin TR: Trends in tuberculosis-United States, 2013. MMWR Morbidity and mortality weekly report 2014, 63(11):229.

13. Frieden TR, et al.: The emergence of drug-resistant tuberculosis in New York City, . New England journal of medicine, 1993, 328(8):521-526.

14. Johnston JC, et al: Treatment outcomes of multidrug-resistant tuberculosis: a systematic review and meta-analysis, . PloS one 2009, 4(9):e6914.

15. Yanina Balabanova, Birute Radiulyte, Edita Davidaviciene: Survival of drug resistant tuberculosis patients in Lithuania: retrospective national cohort study, . BMJ 2011. 
16. Ekaterina V. Kurbatova, Victoria M. Gammino, Jaime Bayona, Mercedes Becerra.: Predictors of poor outcomes among patients treated for multidrug-resistant tuberculosis at DOTS-plus projects, . ELSEVIER, 2012.

17. Kliiman K, Altraja A: Predictors of poor treatment outcome in multi-and extensively drug-resistant pulmonary TB, . European Respiratory Journal, 2009, 33(5):10851094.

18. Kocfa Chung-Delgado, Alejandro Revilla-Montag: Mortality among MDR-TB Cases: Comparison with Drug-Susceptible Tuberculosis and Associated Factors, . PloS ONE 2014, 10(3).

19. Theodros Getachew, A.B.a.B.W.: Survival and Predictors of Mortality among Patients under Multi-Drug Resistant Tuberculosis Treatment .In Ethiopia: St. Peter's Specialized Tuberculosis Hospital, Ethiopia, . International Journal of Pharmaceutical Sciences and Research 2017.

20. Vladimir Milanov, Dennis Falzon, Mariya Zamfirova, Tonka Varleva, Elizabeta Bachiyska, Antoniya Koleva, Masoud Dara: Factors associated with treatment success and death in cases with multidrug-resistant tuberculosis in Bulgaria, . 20092010ELSEVIER, 2015.

21. Chilongola, E.W.M.a.J.O.: Predictors for Mortality among Multidrug-Resistant Tuberculosis Patients in Tanzania, . Hindawi, 2017.

22. Federal Ministry of Health (FMOH): Tuberculosis, leprosy and TB/HIV prevention and control program (manual). Addis Ababa. Ministry of Health, 2008.

23. Federal Ministry of Health (FMOH): The Global MDR-TB and XDR-TB Response Plan 2007-2008. Geneva 2007.

24. Sharma SK, Mohan A: Multidrug-resistant tuberculosis Indian J Med Res 2004, $120(4): 354-376$.

25. World Health Organization (WHO): drug resistance surveillance and response,. 2014.

26. World Health Organization (WHO): Global Tuberculosis Control Report. Report 2009. 
27. Tadele Girum, Samuel Dessu: Survival Status and Treatment Outcome of Mult-drug Resistant Tuberculosis (MDR-TB) among Patents Treated in Treatment Initiation Canters (TIC) in South Ethiopia, . Medical and Health science research, 2017.

28. Velayati AA, et al.: Emergence of new forms of totally drug-resistant tuberculosis bacilli: super extensively drug-resistant tuberculosis or totally drug-resistant strains in Iran,. Chest Journal, 2009, 136(2):420-425.

29. AW Dreyer: National Tuberculosis Management, Guidelines, Department of Health, RSA. 2014.

30. Nimesh Amrith Jayasuriya, Nayanathara Iddamalgoda, Kathy Derore, et.al: Food Security and Nutrition among the Tuberculosis infected patient, Guideline, . 2013.

31. Jennifer Furin: Treatment with a Focus on Co-Morbidities and Adherence, Guideline, . 2014.

32. Lönnroth K, Harries AD: Addressing the global diabetes epidemic to improve tuberculosis prevention and care - from evidence to policy and practice,. Lancet Diabetes and Endocrinology, 2014, 2:730-397.

33. Sun YN, Wang GJ, Zhen XA, Liu ZF, Harley D, Hall G, Vally H, Sleigh A: The long term(9-year)survival of multidrug-resistant tuberculosis patients compared to nonmultidrug-resistant tuberculosis patients in Henan province BMJ open, 2013, 34(2):133-136.

34. World Health Organization (WHO): A Ministerial Meeting of High M/XDR TB Burden Countries in Beijing,. 2009.

35. F. M, Franke S.C.A, Jaime Bayona, Fernando Arteaga, Eda Palacios, Karim Llaro, Sonya S. Shin, Mercedes C. Becerra, Megan B. Murray, Carole DMitnick: Mitnick, Risk Factors and Mortality Associated with Default from Multidrug-Resistant Tuberculosis Treatment,. 2008.

36. Mohsen A. Gadallah, Mervat Rady, Essam El-Moghazy, Magdy Fawzy, Sahar Khalil Kandil: Prognostic factors of treatment among patients with multidrug-resistant tuberculosis in Egypt, . 2015.

37. Omar Salad Elmi, HH., Abdullah S, Mat Zuki Mat Jeab, Zilfalil Ba, Nyi Naing: Treatment Outcomes of Patients with Multidrug-Resistant Tuberculosis (MDR- TB) 
Compared with Non-MDR-TB Infections in Peninsular Malaysia,. Malays J Med SC 2016, 23(4):17-25.

38. Kulchavenya E: Extrapulmonary tuberculosis: are statistical reports accurate? There Adv Infect Dis, . 2014, 2(2):61-70.

\section{Figures}

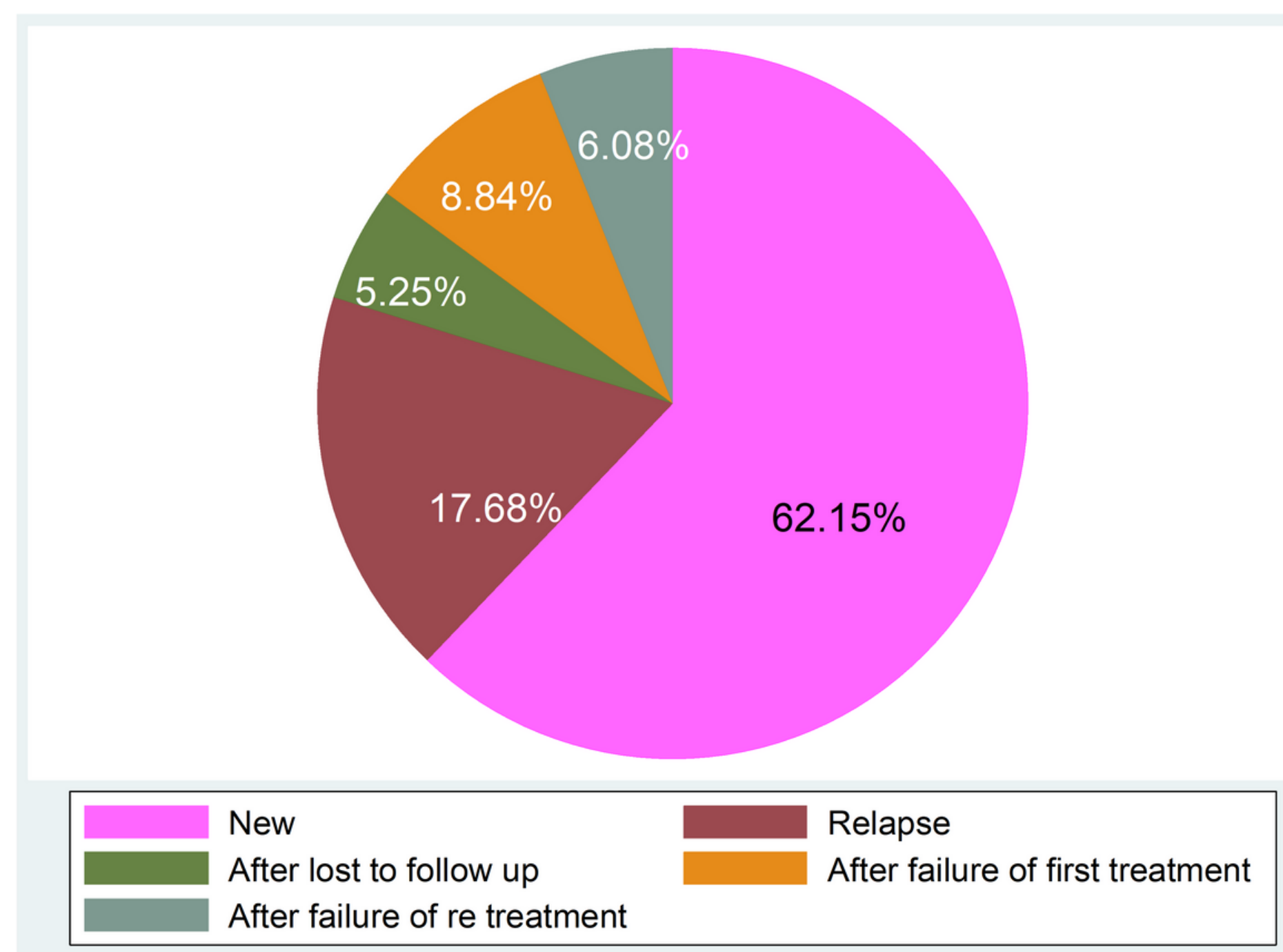

Figure 1

Tuberculosis registration group of patients with DRTB in Eastern and East-Central Ethiopia, from September 2012 to 30th August 2017 


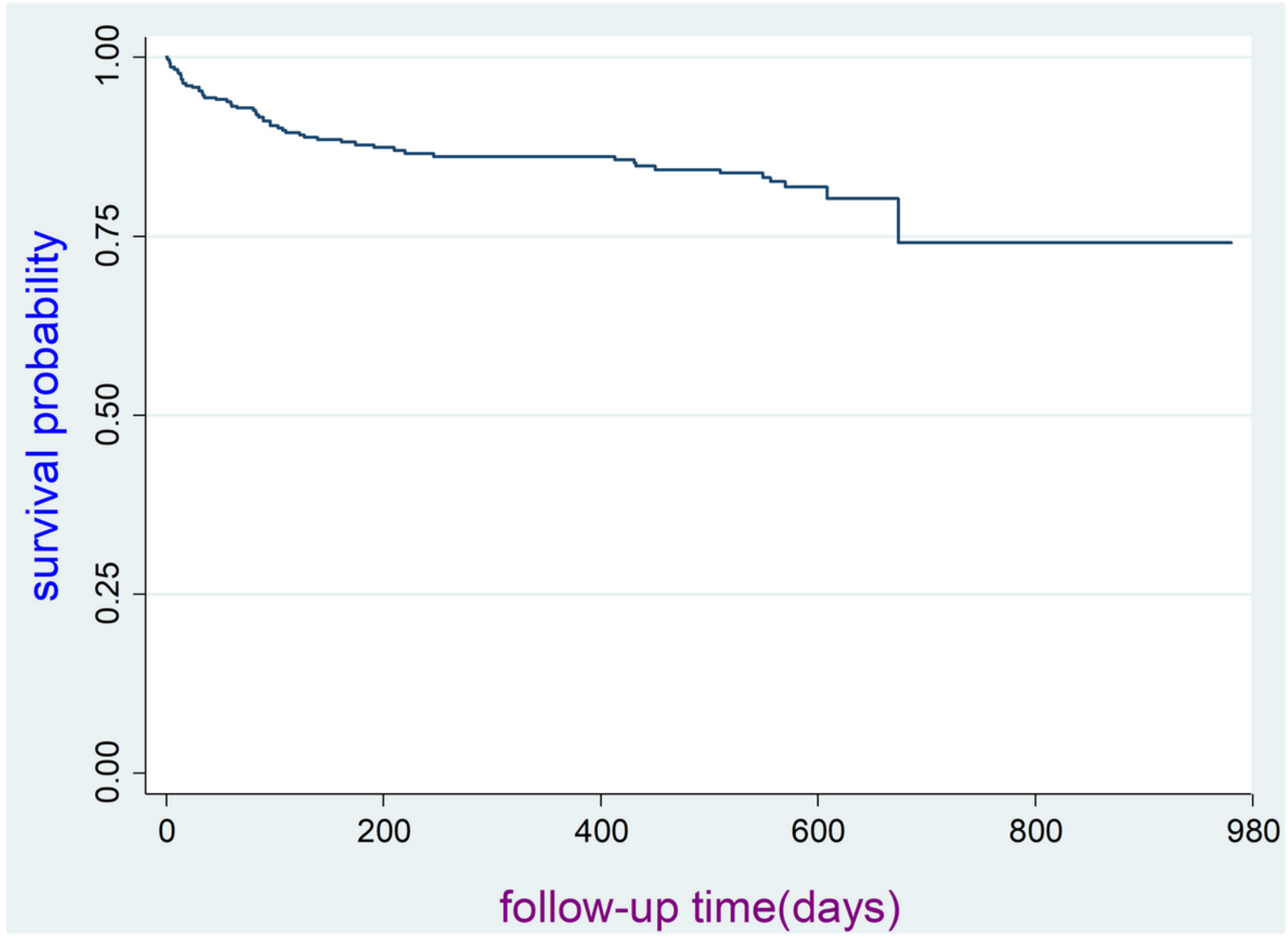

Figure 2

The overall survival status of DRTB in Eastern and East-Central Ethiopia, from September 2012 to 30th August 2017 


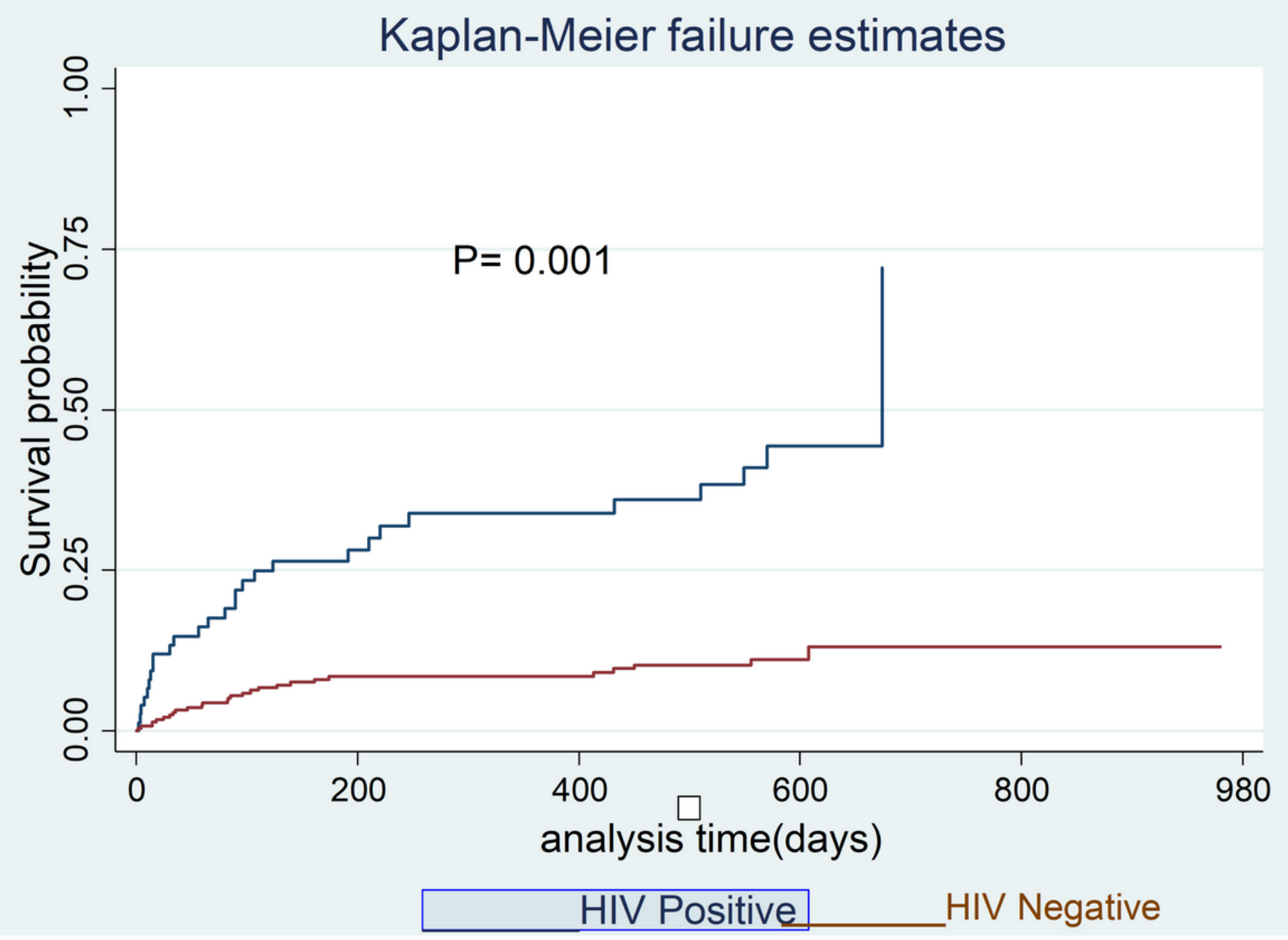

Figure 3

The Hazard of death among adult DRTB patients by their HIV status in Eastern and East Central Ethiopia, 2012-2018

\section{Supplementary Files}

This is a list of supplementary files associated with this preprint. Click to download.

- supplementaryfile.docx

- supp.docx 